\title{
Study of Electronic Properties of GaAs Semiconductor Using Density Functional Theory
}

\author{
Fikri Abdi Putra, ${ }^{1, a}$, Endhah Purwandari ${ }^{1}$ and Bintoro S. Nugroho ${ }^{2}$ \\ ${ }^{1}$ Department of Physics, Faculty of Mathematics and Natural Sciences, Universitas Jember, \\ Jalan Kalimantan No. 37, Jember 68121, Indonesia \\ ${ }^{2}$ Physics Department, Faculty of Mathematics and Natural Sciences, Universitas Tanjungpura, \\ Jl. Prof. Dr. H. Hadari Nawawi, Pontianak, Indonesia \\ afikriabdi21@gmail.com
}

\begin{abstract}
The properties of GaAs material in zinc blende type was calculated using Hiroshima Linear Plane Wave program based on the Density Functional Theory. This calculation aims to determine electronic properties of GaAs material are based on Density of States and energy band structure. This simulation's results are DOS shows that hybridization of $s$ orbital of $\mathrm{Ga}$ with $s$ orbital of As provides covalent properties. The simulation of energy band structure from GaAs material indicates that semiconductor properties of GaAs is direct band gap. The energy band gap results obtained for GaAs is $0.80 \mathrm{eV}$. The computational result of the energy band gap calculation form HiLAPW has better accuracy and prediction with good agreement within reasonable acceptable errors when compared to some other DFT programs and the results of the experimental obtained.
\end{abstract}

Keywords: DFT, HiLAPW, GaAs, Electronic Properties, Density of States, Energy Band Structure, Energy Bandgap

\section{Introduction}

The study of materials applied as electronic materials, magnetic materials, spintronic materials, optical materials, etc. has a very important role in the development of science and technology. A widely studied material in this regard is Gallium Arsenide (GaAs). Analysis of its characteristics is used for the development of electronics and instrumentation technology. The performance of GaAs materials selected to be applied as optical materials for lasers [1], detectors and photovoltaics [2], also as substrate materials [3,4], are influenced by their electronic properties. The electronic properties could be analyzed from the characteristics of the density of state (DOS) and energy band diagram, as well as the band gap of the material.

The calculation of density of state (DOS) and energy band of GaAs have been reported by Rany and Kumar [5] using Density Functional Theory implemented by SIESTA code, in local density approximation with exchange correlation parameterized by Perdew and Zunger. The GaAs energy band gap, which was simulated using the zincblende structure, was $0.4 \mathrm{eV}$. Experimentally, the measurement of the energy gap was carried out by Kusch et al using Raman Resonance Spectroscopy. At room temperature and $0 \mathrm{~K}$, the energy gaps of GaAs with a zincblende crystal structure were obtained at $1.425 \mathrm{eV}$ and $1.519 \mathrm{eV}$, respectively [6]. In this paper, we perform the calculation of band gap of GaAs zincblende type based on the band structure using DFT method through HiLAPW program package. It is designed to perform bandstructure calculations based on the density functional theory (DFT), with some features including scalar-relativistic spin-polarized calculations within the local (spin) density 
approximation (LSDA); all-electron self-consistent calculations; total-energy and atomic-force calculations for determining the equillibrium structure and phonons; electron density-of-states (DOS) calculations; and also for 2D or 3D drawing of electron density and potential function calculations [7]. The energy gap of GaAs obtained have been compared with other DFT methods and also with experiments as validation result.

\section{Theoretical Background}

GaAs is an alloy semiconductor material consisting of Gallium (Ga) of group III and Arsenide (As) of group V [8]. GaAs has a space group F-43m [9] with zinc blende type structure [10] as shown in Figure 1.

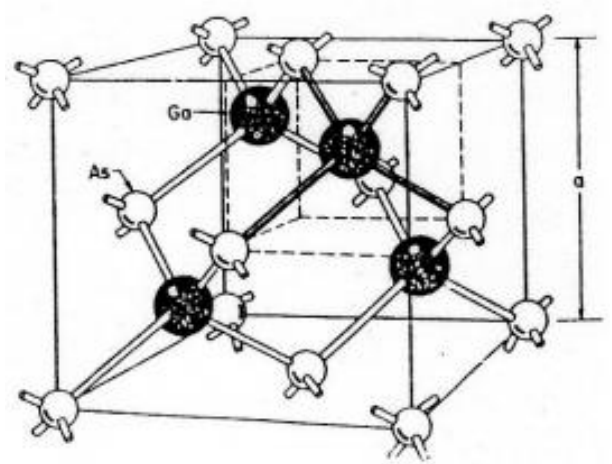

Figure 1. The crystal structure of GaAs [10,11]

This material is classified as a semiconductor, which is widely studied and developed as nano materials products [13] as well as the substrate for other materials [3,4,12]. Density Functional Theory (DFT) is a numerical method based on electron density which replaces the electron wave function $[14,17]$ developed with the approach of Hohenberg-Kohn theory [15] and outlined in the Kohn-Sham equation [16].

\section{Materials and Methods}

This material simulation program uses the DFT method of HiLAPW. Hiroshima Linear Plane Wave (HiLAPW) is non-open-source simulation program developed by Prof. Tamio Oguchi (Japan) from the theory group of Hiroshima University on the Linux operating system using the Fortran code. The program is used to calculate Density of States (DOS) and energy band structure until it reaches a self-consistent state is means a state when electron density at the end of the calculation is close to the input of the previous electron density so that the ground state condition is obtained [17]. The calculation is conducted by iteration process starting with guessing the electron density, which is a function formed by the wave function of electrons. The calculation is continued by calculating the Hartree potential and the potential exchangecorrelation to obtain a solution of the calculation in the form of Schrödinger equation as

$$
\left[-\frac{1}{2} \nabla^{2}+V_{\text {ext }}(\vec{r})+V_{H}(\vec{r})+V_{x c}(\vec{r})\right] \phi_{i}(\vec{r})=\varepsilon_{i} \phi_{i}(\vec{r})
$$


Solving Equation (1) produces a new wave function that forms the density of new electrons. If the density is equal to or close enough to the initial input density, a self-consistent condition is obtained. If the condition has not been reached, the calculation is repeated with the input density of the newly obtained density value. Inputs in the HiLAPW DFT calculation are lattice parameters, space group, atomic position in unit cells, k-point mesh. Details of some parameters of GaAs structure are listed in Table 1.

Table 1. Parameter of GaAs material zinc blende structure

\begin{tabular}{ccccccc}
\hline Space & \multicolumn{3}{c}{ Lattice parameters } & \multicolumn{2}{c}{ Vector } & k-point \\
Group & \multicolumn{2}{c}{ Ga } & As & mesh \\
\hline & & & & & \\
F-43m & $5.652 \dot{A}$ & $5.652 \dot{A}$ & $5.652 \dot{A}$ & 0 & 0.25 & \\
& $90^{\circ}$ & $90^{\circ}$ & $90^{\circ}$ & 0 & 0.25 & 444 \\
& & & & 0 & 0.25 & \\
\hline
\end{tabular}

The electron density simulation uses the JOB-SCF shell-script containing input parameters (sets.in) and SCF (law.in) control along with other supporting files. This simulation produces output (wave.out) that is used for DOS simulation and energy band structure by converting it into input (wavin). The next step of DOS simulation using JOB-DOS contains input (doss.in) and wavin from SCF calculation to get tdos.ps and pdos.ps. The file is plotted with PSP commands using GNUPLOT to generate TDOS and PDOS curve. Wavin file from the SCF simulation and input (kpts.in) is used for JOB-EK simulation generate eigen-energy value. This value is used to obtain results in the form of eigen-state. This result is used to plot energy band structure with PSP command using GNUPLOT to obtain energy band structure curve. DOS and energy band structure plot results will later be used for the analysis of the results. After the analysis of curve is carried out, a conclusion will be obtained.

\section{Results and Discussion}

\section{Density of State (DOS) of GaAs}

The calculation of Density of State (DOS) in the Total Density of States (TDOS) and Partial Density of States (PDOS) are completed using the JOB-DOS shell script and plotted with GNUPLOT to obtain TDOS and PDOS curve. The curve of TDOS is analyzed based on the density distribution of the electron states that contribute to GaAs material [18], while the curve of PDOS is analyzed based on the density of each the orbital states of constituent atomic electrons that contribute to GaAs material. 


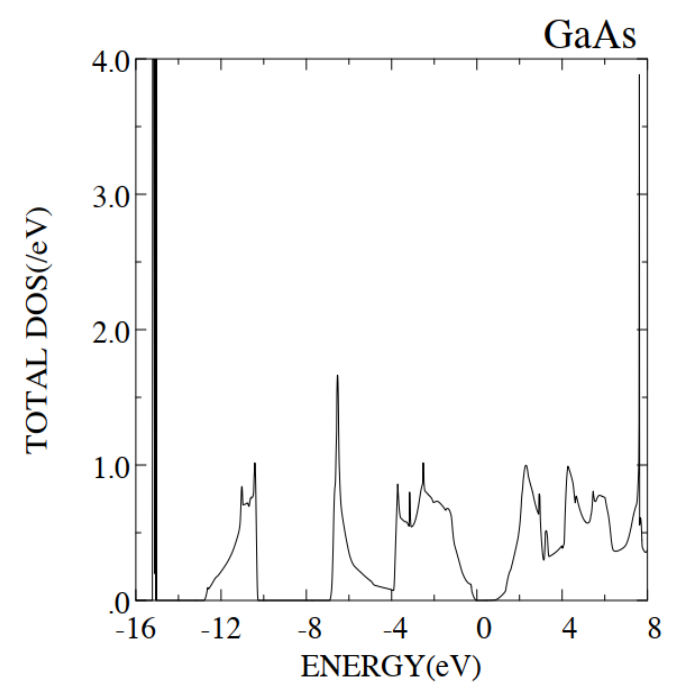

(a)

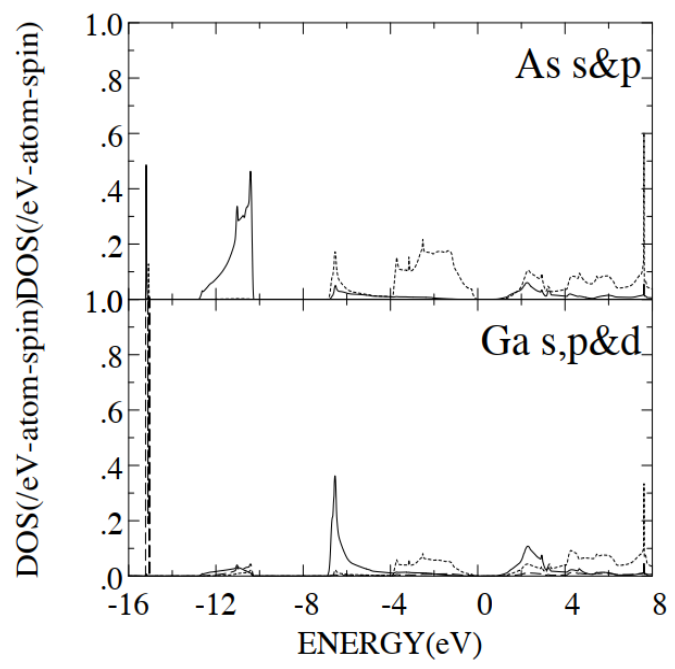

(b)

Figure 2. Curve of DOS on GaAs material (a) TDOS and (b) PDOS

The curves of DOS consisting of TDOS and PDOS is shown in Figure 2 with the $x$-axis indicating the energy level in (eV) units and the y-axis indicating the magnitude of the states of TDOS and PDOS in (states/eV) units. It can be seen in Figure 2 (a) that the energies ranging from $0 \mathrm{eV}$ to $16 \mathrm{eV}$ perform the valence band, while the conduction band is mainly distributed between $0 \mathrm{eV}$ and $8 \mathrm{eV}$. The zero energy indicates the Fermi level of GaAs. Figure 2 (b) exhibit the partial density of state of each element. The solid line defines the s-orbital, as well as the dash line represents the $p$-orbital. The highest state valence band is possessed by the PDOS of As $s$, ranging from $-13 \mathrm{eV}$ to $-10 \mathrm{eV}$. The lowest state of valence bands is entirely from $\mathrm{Ga} d$, where the peaks are less noticeable. The upper most group of valence bands is clearly dominated by As $p$, with a significant overlap with $\mathrm{Ga} s$ and a smaller contribution from $\mathrm{Ga} p$. The conduction band in PDOS curve shows that $\mathrm{Ga}$ and As have overlapping states as evidenced by similar states with the same high energy ranges. Based on this, the states that formed in TDOS have a widening of states with a high energy range. The conduction band overlap between orbital Ga $s$ and orbital As $p$ indicate an hybridization characteristics, where their interactions represent covalent bonds.

\section{Energy Band Structure of GaAs}

The curve of the energy band structure is related to the permissible energy range and the not allowed electron wave function [19]. This energy range describes electronic states [20] means that the energy band structure is correlated with DOS shown in Figure 2, where the states on the TDOS curve denote an energy band. The energy band structure is calculated via the JOBBS shell script and plotted using GNUPLOT program to obtain the energy band structure curve shown in Figure 3. 


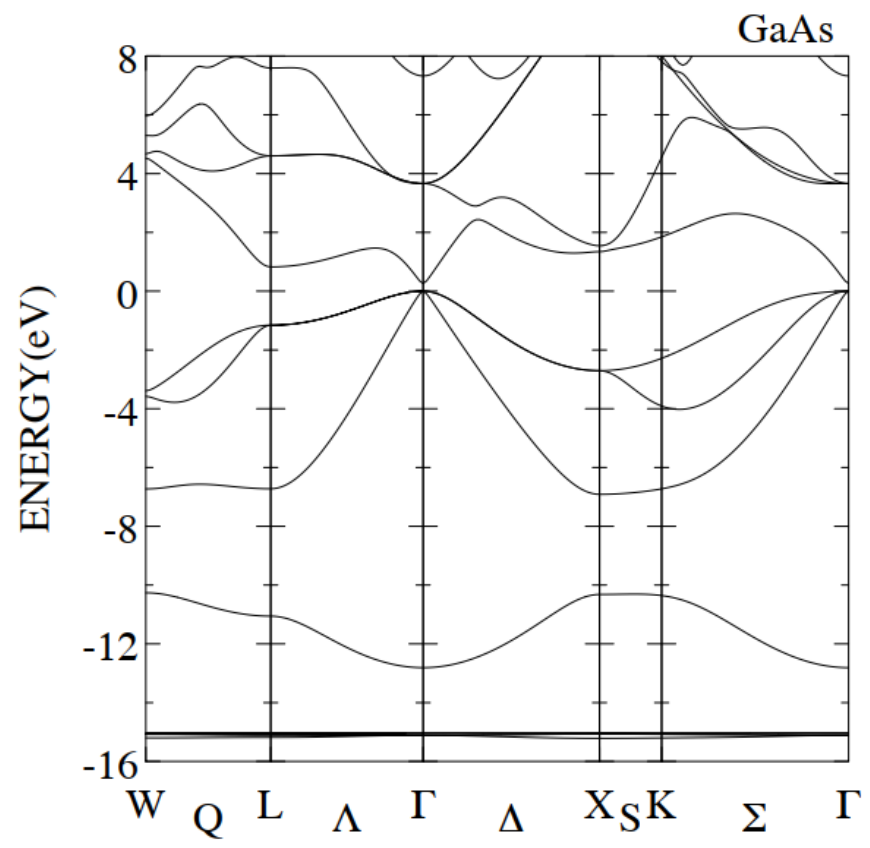

Figure 3. Curve of the energy band structure of GaAs material

The curve of the energy band structure of GaAs material has a y-axis indicating energy in (eV) and the $\mathrm{x}$ - axis indicating k-points of the Brillouin zones symmetry points. Based on the structure energy band, there is an energy band is flat in valence band. These energy band is poorly dispersed, which results in very low electron states formed from contribution of orbitals $d$ shown in PDOS states in Figure 2. The energy band of the orbital $d$ or orbital $f$ is an energy band localized to the atomic nucleus so that the resulting band is less dispersed, while the dispersed band of energy in the form of parabolic (curly) is energy band of orbital $s$ and orbital $p$ [21]. Based on the energy band structure shows that the maximum valence band and the minimum conduction band are located on the same symmetry point $\Gamma$ indicates that the bandgap of this material is direct bandgap. Comparison of the energy bandgap value of this study with the results of previous simulations and experiment results is performed by Table 2.

Table 2. Comparison of the energy bandgap value of this study with the results of previous simulations and experiment results

\begin{tabular}{|c|c|c|c|}
\hline Materials & Energy bandgap (eV) & Method & Reference \\
\hline \multicolumn{4}{|c|}{ A. Computation } \\
\hline GaAs & 0.8 & DFT (HiLAPW), zinc blende, LDA & - \\
\hline GaAs & 0.099 & DFT (WIEN2k), zinc blende, LDA & [22] \\
\hline GaAs & 0.4 & DFT (SIESTA), zinc blende, LDA & [5] \\
\hline GaAs & 0.38 & DFT (FHI-Aims), zinc blende, LDA & [20] \\
\hline \multicolumn{4}{|c|}{ B. Experiment } \\
\hline GaAs & 1.519 & $\begin{array}{c}\text { Raman Measurements, zinc } \\
\text { blende nanowires }\end{array}$ & [6] \\
\hline
\end{tabular}


The calculation of the energy bandgap using the HiLAPW program is obtained of $0.8 \mathrm{eV}$. This amount is greater than other calculation result $[5,20,22]$. While the energy bandgap value produced from the experiment result by [6] is $1.519 \mathrm{eV}$ and the reference from [19] is $1.52 \mathrm{eV}$. Based on the comparison of these data, it can be concluded that the energy gap calculation using HiLAPW code packages is more accurate and has a better approach to experimental results than other DFT programs.

\section{Conclusions}

The calculation of Density of States (DOS) and energy band structure has been carried on zinc blende type of GaAs with the HiLAPW program of DFT method. The results of DOS calculations showed an interaction between orbital $s \mathrm{Ga}$ and orbital $p$ As formed hybridization, so giving covalent characteristic to semiconductor. The results of calculation on the energy band structure indicate the presence of semiconductor properties that match the results of previous experiments and research on GaAs material shows that GaAs is a direct bandgap semiconductor with an energy bandgap value of $0.80 \mathrm{eV}$. The HiLAPW program has computationally demonstrated good accuracy and prediction on the calculation of energy band structure and energy band gap.

\section{Acknowledgement}

Thanks to Prof. Tamio Oguchi who has provided training on DFT computing in the HiLAPW program at the Computational Physics Laboratory, Department of Physics, Universitas Jember on $30^{\text {th }}$ July $-1^{\text {st }}$ August 2018 during Guest Lecture "DFT, HILAPW and Its Applications in Material Research" organized by Dr. Artoto Arkundato (Head of Computational Physics Laboratory).

\section{References}

[1] M. Sharmin, S. Choudhury, N. Akhtar, and T. Begum, 2012, Optical and Transport Properties of p-Type GaAs, Journal of Bangladesh Academy of Sciences, volume 36, no. 1, page 97-107, doi: 10.3329/jbas.v36i1.10926.

[2] P. Cells and U. Kingsley, 2016, Calculation of the Electronic Properties of Gallium Arsenide ( $\mathrm{GaAs}$ ) Calculation of the Electronic Properties of Gallium Arsenide ( GaAs ) Semiconductors for Photovoltaic Cells, volume 5, no. 9, page 99-104.

[3] E. C. Larkins and J. S. Harris, 1995, Molecular Beam Epitaxy of High-Quality $\mathrm{GaAs}$ and $\mathrm{AlGaAs}$, in Molecular Beam Epitaxy Applications to Key Materials, R. F. C. Farrow, Ed. New Jersey: Noyes Publication, page 114-274. doi: https://doi.org/10.1016/B978-081551371-1.50004-4.

[4] D. A. Jameel et al., 2020, Investigation of the effects of GaAs substrate orientations on the electrical properties of sulfonated polyaniline based heterostructures, Applied Surface Science, volume 504, page 144315, doi: 10.1016/j.apsusc.2019.144315.

[5] A. Rani and R. Kumar, 2015, Structural and electronic properties of GaAs and 


\section{Computational and Experimental Research in Materials and Renewable Energy (CERiMRE) Volume 4, Issue 2, page 94-101 elSSN : 2747-173X}

Submitted : August 20, 2021

Accepted : October 25, 2021

Online : November 24, 2021

DOI : $10.19184 /$ cerimre.v4i2.28375

GaP semiconductors, AIP Conference Proceedings, volume 1661, doi: 10.1063/1.4915369.

[6] P. Kusch, S. Breuer, M. Ramsteiner, L. Geelhaar, H. Riechert, and S. Reich, 2012, Band gap of wurtzite GaAs: A resonant Raman study, Physical Review B Condensed Matter and Materials Physics, volume 86, no. 7, page 1-5, doi: 10.1103/PhysRevB.86.075317.

[7] T. Oguchi, Hilapw Project, 2003. http://www.cmp.sanken.osakau.ac.jp/ oguchi/HiLAPW/index.html (accessed Jan. 15, 2020).

[8] H. Arabi, A. Pourghazi, F. Ahmadian, and Z. Nourbakhsh, 2006, First-principles study of structural and electronic properties of different phases of GaAs, Physica B: Condensed Matter, volume 373, no. 1, page 16-22, doi: 10.1016/j.physb.2005.10.130.

[9] A. Mujica, A. Rubio, A. Muñoz, and R. J. Needs, 2003, High-pressure phases of group-IV, III-V, and II-VI compounds, Reviews of Modern Physics, volume 75, no. 3, page 863-912, doi: 10.1103/RevModPhys.75.863.

[10] J. Singleton, 2001, Band Theory and Electronic Properties of Solids Band 2 Oxford Master Series in Condensed Matter Physics. page 157-158.

[11] S. M. Sze, 2002, Semiconductor Device: Physics and Technology, 2nd ed. John Wiley \& Sons, Inc.

[12] J. Chawich et al., 2020, Deposition and characterization of ZnO thin films on GaAs and Pt/GaAs substrates, Materials Chemistry and Physics, volume 247, doi: 10.1016/j.matchemphys.2020.122854.

[13] L. Liu, Y. Diao, and S. Xia, 2019, High-performance GaAs nanowire cathode for photon-enhanced thermionic emission solar converters, Journal of Materials Science, volume 54, no. 7, page 5605-5614, doi: 10.1007/s10853-018-03231-8.

[14] S. Cottenier, 2002, Density Functional Theory and the Family of (L)APWmethods: a step-by-step introduction. Belgium: Instituut voor Kern- en Stralingsfysica, K.U.Leuven. [Online]. Available: http://www.wien2k.at/reg user/textbooks

[15] P. Hohenberg and W. Kohn, 1964, Inhomogeneous Electron Gas, Physical Review, volume 136, no. 3B, page 864-871, doi: 10.1103/physrev.136.b864.

[16] W. Kohn and L. J. Sham, 1965, Self-Consistent Equations Including Exchange and Correlation Effects, Physical Review, volume 140, no. 4A, page A1133A1138, doi: 10.1103/physrev.140.a1133.

[17] M. M. Richard, 2004, Electronic Structure, Basic Theory and Practical Method. Cambridge: Cambridge University Press.

[18] M. Sach, 1963, Solid State Theory. New York: McGraw-Hill Book Company.

[19] K. Charles, 2004, Introduction to Solid State Physics, 8nd ed. USA: John Wiley \& 
Sons, Inc.

[20] J. A. Owolabi, M. Y. Onimisi, S. G. Abdu, and G. O. Olowomofe, 2016, Determination of Band Structure of Gallium-Arsenide and Aluminium-Arsenide Using Density Functional Theory, Computational Chemistry, volume 04, no. 03, page 73-82, doi: 10.4236/cc.2016.43007.

[21] S. Mohammad, 2013, First-Principles Study on Dilute Magnetic States and Half Metallicity in Chalcopyrite Semiconductors, Osaka University, doi: 10.18910/34052.

[22] N. N. Anua, R. Ahmed, M. A. Saeed, A. Shaari, and B. U. Haq, 2012, DFT investigations of structural and electronic properties of Gallium Arsenide (GaAs), AIP Conference Proceedings, volume 1482, page 64-68, doi: 10.1063/1.4757439. 